\title{
Magnetic localization in mixed-valence manganites
}

\author{
M. Viret, ${ }^{*}$ L. Ranno, and J. M. D. Coey \\ Physics Department, Trinity College, Dublin 2, Ireland \\ (Received 24 September 1996; revised manuscript received 25 November 1996)
}

\begin{abstract}
The metal-insulator transition is mixed-valence manganites of the $\left(\mathrm{La}_{0.7} \mathrm{Ca}_{0.3}\right) \mathrm{MnO}_{3}$ type is ascribed to a modification of the spin-dependent potential $J_{H} \mathbf{s} \cdot \mathbf{S}$ associated with the onset of magnetic order at $T_{C}$. Here $J_{H}$ is the on-site Hund's-rule exchange coupling of an $e_{g}$ electron with $s=1 / 2$ to the $t_{2 g}$ ion core with $S$ $=3 / 2$. Above $T_{C}$, the $e_{g}$ electrons are localized by the random spin-dependent potential and conduction is by variable-range hopping. Over the whole temperature range, the resistivity varies as $\ln \left(\rho / \rho_{\infty}\right)$ $=\left[T_{0}\left\{1-\left(M / M_{S}\right)^{2}\right\} / T\right]^{1 / 4}$, where $M / M_{S}$ is the reduced magnetization. The temperature and field dependence of the resistivity deduced from the molecular-field theory of the magnetization reproduces the experimental data over a wide range of temperature and field. [S0163-1829(97)04513-X]
\end{abstract}

Interest in mixed-valence manganites of the $\left(\mathrm{La}_{0.7} \mathrm{Ca}_{0.3}\right) \mathrm{MnO}_{3}$ type has revived ${ }^{1}$ with the observations of large negative magnetoresistive effects, ${ }^{2,3}$ especially in suitably annealed thin films. ${ }^{4}$ The magnetoresistance is greatest in the vicinity of the Curie point $T_{C}$ of ferromagnetic compositions which exhibit "metallic" (temperatureindependent) conduction at low temperatures and thermally activated conduction above $T_{C}$. These compositions have a structure which is a variant of the cubic perovskite cell where the $\mathrm{Mn}-\mathrm{O}$ bond lengths are unequal and $\mathrm{Mn}-\mathrm{O}-\mathrm{Mn}$ bond angles differ from $180^{\circ} .5^{5}$ Their electronic properties are related to electron hopping among the Mn ions in octahedral sites; metallic conductivity and ferromagnetism are closely related and are generally interpreted in terms of the doubleexchange mechanism. ${ }^{6}$ A spin-polarized $\sigma^{*}$ conduction band of mainly $3 d\left(e_{g} \uparrow\right)$ character $^{7}$ is supposed to be responsible for the "metallic" character of the current transport below $T_{C} \cdot{ }^{8}$ The $\mathrm{Mn}^{3+}$ ion has one $e_{g}$ electron, whereas the $\mathrm{Mn}^{3+}$ ion has none. When the concentration of the divalent $A$-site cation (Ca, for example) is 0.3 , the occupancy of the $\sigma^{*}$ band is 0.7 , which corresponds to the strongest ferromagnetism and the greatest magnetoresistance.

Electron transfer with spin memory is an essential ingredient for an understanding of the transport properties of mixed-valence manganites, but something more is needed to account for the metal-insulator transition near the Curie point. ${ }^{9}$ The change of conduction regime below $T_{C}$ appears to be brought about by the onset of ferromagnetism. As temperature decreases, the magnetization increases and the resistivity drops. Resistivity has been reported to vary like $\left[1-\left(M / M_{S}\right)^{2}\right]$, as in conventional giant magnetoresistance (GMR) systems, ${ }^{10}$ but others find an exponential dependence $^{11} \ln (\rho) \sim-M / M_{S}$. Here we propose the concept of magnetic localization to relate the resistivity at any temperature or applied field to the local magnetization, evaluated in the molecular field approximation. The model involves variable range hopping and goes beyond the purely phenomenological parallel conduction model of Nunez-Reiguero and Kadin. $^{12}$

We previously observed an impressive 11 orders of magnitude span for the residual resistivities $\rho_{0}$ of different compounds ${ }^{13}$ made by substituting different $A$-site cations for $\mathrm{La}$ and $\mathrm{Ca}$, while keeping the same stoichiometry, lattice parameter, and band filling. In fact, using Mott's criterion for metallic conductivity $\rho_{0}<1 \mathrm{~m} \Omega \mathrm{cm},{ }^{14}$ only $\left(\mathrm{La}_{0.7} \mathrm{Sr}_{0.3}\right) \mathrm{MnO}_{3}$, the compound with the highest $T_{C}$ and the smallest magnetoresistance, can be properly considered as metallic at low temperature. Gupta et al. ${ }^{15}$ have demonstrated that the residual resistivity increases rapidly with decreasing crystallite size in polycrystalline films. The temperature variation below $T_{C}$ is also much too large to be conventionally accounted for by electron-phonon scattering. We previously suggested ${ }^{13}$ that the electrons may be localized in ferromagnetic wave packets and the current at low temperature is transported by zero-point hopping of the carriers between these packets across barriers which are associated with canted spins. Now we extend the idea of magnetic potential barriers to electron transport to explain the electrical behavior over the whole temperature range.

Above $T_{C}$, some groups report semiconducting behavior $\ln \left(\rho / \rho_{\infty}\right)=E_{a} / k T,{ }^{16}$ with activation energy of the order of 0.2 $\mathrm{eV}$, while others find their data can be well fitted by Mott's variable-range-hopping (VRH) model $\ln \left(\rho / \rho_{\infty}\right)$ $=\left(T_{0} / T\right)^{1 / 4} \cdot{ }^{13,17,18}$ We have measured resistivity vs temperature characteristics for a wide range of samples which exhibit a metal-insulator transition. The $T^{-1}$ law, which would be characteristic of nearest-neighbor hopping or activation to a mobility edge, does not fit the data. The $T^{-1 / 2}$ law, which is characteristic of variable-range hopping with a soft gap due to electron correlations, ${ }^{19}$ leads acceptable fits. But we find that the data best follow a $T^{-1 / 4}$ law above $T_{C}$, supporting the VRH model as the mechanism for current transport in the manganites. In Fig. 1 we have plotted $T_{0}$ values for a wide range of substituted manganites with $x=0.3$. Samples were either as-deposited films or bulk ceramics. Above $T_{C}$, the curves are flat indicating that $T_{0}$ is independent of $T$ in that range. Two notable exceptions are $\left(\mathrm{Sm}_{0.7} \mathrm{Ba}_{0.3}\right) \mathrm{MnO}_{3}$ and $(\mathrm{LaMn})_{0.95} \mathrm{O}_{3}$ (Fig. 1), both of which show a monotonic increase in resistivity below $T_{C}$. The resistivity of the cationdeficient compounds $(\mathrm{LaMn})_{0.95} \mathrm{O}_{3}$, was best fitted with a simply activated semiconducting law. These are the object of study of another publication. ${ }^{20}$ For now, let us examine the conduction process in the majority of our ferromagnetic manganites. 


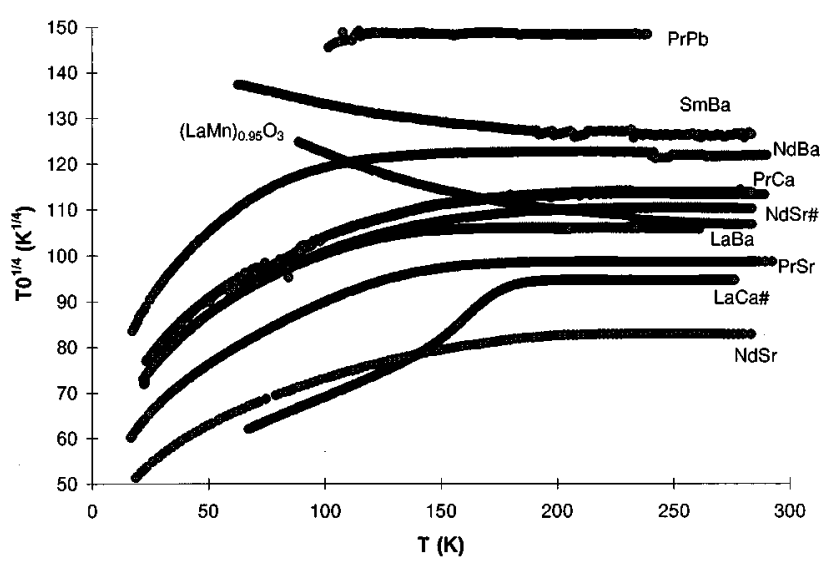

FIG. 1. Plot of the VRH parameter $T_{0}$ as a function of temperature a series of $A_{0.7} B_{0.3} \mathrm{MnO}_{3}$ compounds with different $A$ and $B$ cation pairs as indicated on the curves. Here \# is for thin films, the others being polycrystalline bulk samples.

Originally, the VRH theory ${ }^{21}$ was developed to explain electron transport in doped semiconductors where electrons occupying hydrogenic orbitals with wave functions $\psi$ $=\psi_{0} \exp (-\alpha r)$ are localized by potential fluctuations associated with the dopant. There is a competition between the potential energy difference and the distance electrons can hop. This is reflected in the expression of the hopping rate to a site at a distance $R$ where the energy of the carrier is $\Delta E$ higher than at the origin:

$$
\gamma=\gamma_{0} \exp (-2 \alpha R-\Delta E / k T) .
$$

A sphere of radius $R$ contains $\frac{4}{3} \pi R^{3} / v$ sites, where $v$ is the lattice volume per manganese ion, $5.7 \times 10^{-29} \mathrm{~m}^{3}$. The smallest value of $\Delta E$ is therefore $\left[\frac{4}{3} \pi R^{3} N(E)\right]^{-1}$ where $N(E)$ is the density of available states. Minimizing the hopping rate, we find $R=\{9 /[8 \pi \alpha N(E) k T]\}^{1 / 4}$, and substituting this value in Eq. (1) gives an expression for the resistivity $(\rho \sim 1 / \gamma)$ :

$$
\rho=\rho_{\infty} \exp \left\{2.06\left[\alpha^{3} / N(E) k T\right]^{1 / 4}\right\},
$$

which is the Mott expression with

$$
k T_{0}=18 \alpha^{3} / N(E)
$$

Other derivations in the literature yield slightly different prefactors. $^{21,22}$ Electron hopping is always of variable-range type at low temperature where the thermal energy is not great enough to allow electrons to hop to their nearest neighbors. In that case it is more favorable for the electrons to hop farther to find a smaller potential difference. At high temperatures, conduction may be by activation above the mobility edge. In the intermediate range, nearest-neighbor hopping can contribute significantly to the transport. These last two types of motion are simply activated $\left[\ln (\rho) \sim T^{-1}\right]$.

To interpret the data above $T_{C}$ in terms of this model, we need the electronic density of states $N(E)$. Heat capacity measurements at low temperatures lead to values of the Sommerfield coefficient $\gamma_{e}$ corresponding to $N\left(E_{F}\right) \approx$ $4 \times 10^{28} \mathrm{~m}^{-3} \mathrm{eV}^{-1}{ }^{13}$ The number of $e_{g}$ electrons is $0.7 / v$ $=1.2 \times 10^{28} \mathrm{~m}^{-3}$, and hence the occupied part of the $e_{g}$ band is about $0.3 \mathrm{eV}$ wide. Values of $k T_{0}$ (Fig. 1) range from
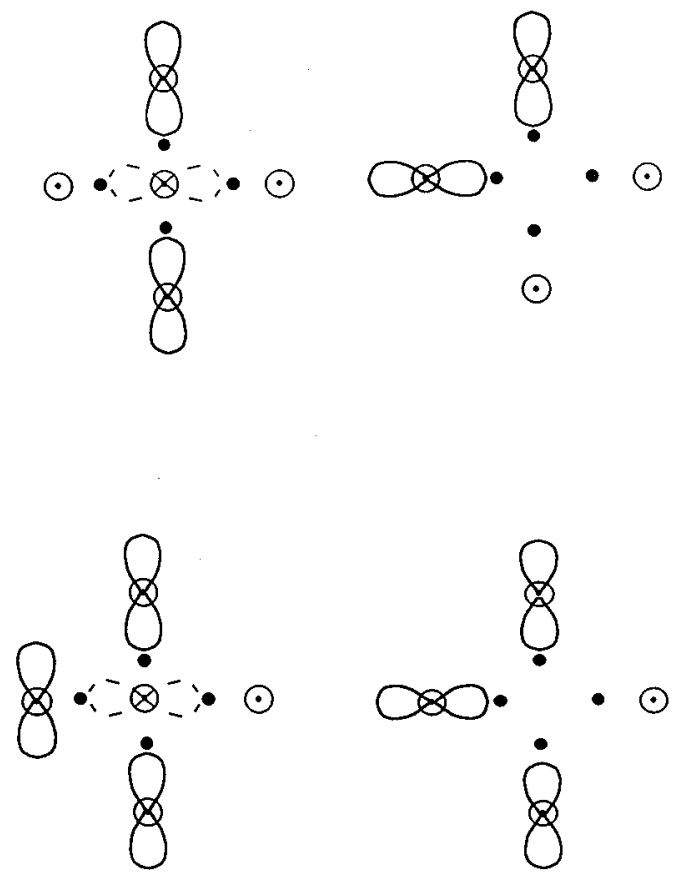

FIG. 2. Examples of nearest-neighbor configurations of a vacant site in a plane which can (left) and cannot (right) receive a $d_{z 2}$ electron. The $\mathrm{Mn}$ atoms are represented by open circles and the oxygens by solid dots.

$220 \mathrm{eV}$ for $\left(\mathrm{La}_{0.7} \mathrm{Sr}_{0.3}\right) \mathrm{MnO}_{3}$ to $43600 \mathrm{eV}$ for $\left(\mathrm{Pr}_{0.7} \mathrm{~Pb}_{0.3}\right) \mathrm{MnO}_{3}$, and the corresponding localization lengths $1 / \alpha$ deduced from Eq. (3) are 0.13 and $0.02 \mathrm{~nm}$, respectively. At room temperature, the values of the average hopping distance are 0.46 and $0.30 \mathrm{~nm}$, respectively. Since the localization must exceed the Mn-Mn distance and the hopping distance should be several times greater, these numbers are incompatible with conventional variable-range hopping.

This unphysical result underlines a fundamental problem: either the density of state decreases drastically above $T_{C}{ }^{23}$ or else the localization mechanism is different. Manganites with $x=0.3$ show little structural change at $T_{C}$, so that any changes in band structure and density of states should be associated with the onset of ferromagnetic order. We propose that a random potential of mainly magnetic origin is responsible for carrier localization above $T_{C}$. This potential is due to the Hund's-rule coupling $-J_{H} \mathbf{S} \cdot \mathbf{S}$ between localized $\mathrm{Mn}$ $t_{2 g}$ ion cores $(S=3 / 2)$ and the spins $s$ of the $e_{g}$ electrons in the $\sigma^{*}$ conduction band.

We write this energy in the form $E_{m}=\frac{1}{2} U_{m}\left(1-\cos \theta_{i j}\right)$, where $\theta_{i j}$ is the angle between the spins of the two Mn ion cores between which the $e_{g}$ electron is hopping and $U_{m}$ $=3 J_{H} / 2$ the splitting between spin-up and spin-down $e_{g}$ bands. Assuming the spin directions of the ion cores are uncorrelated in the paramagnetic state, the probability of finding an angle $\theta_{i j}$ between two ion cores, $p\left(\theta_{i j}\right)$ $=\frac{1}{2} \sin \theta_{i j}$. The probability density of a barrier $E_{m}$ is therefore

$$
p\left(E_{m}\right)=p\left(\theta_{i j}\right) d \theta_{i j} / d E_{m}=1 / U_{m} .
$$

Such a magnetic random potential is significant here because $U_{m}$ is larger than $1 \mathrm{eV}$. A lower limit on $U_{m}$ is set by the fact that $\mathrm{Mn}^{3+}$ is in a high-spin state so that $U_{m}>\Delta$, where $\Delta$ 

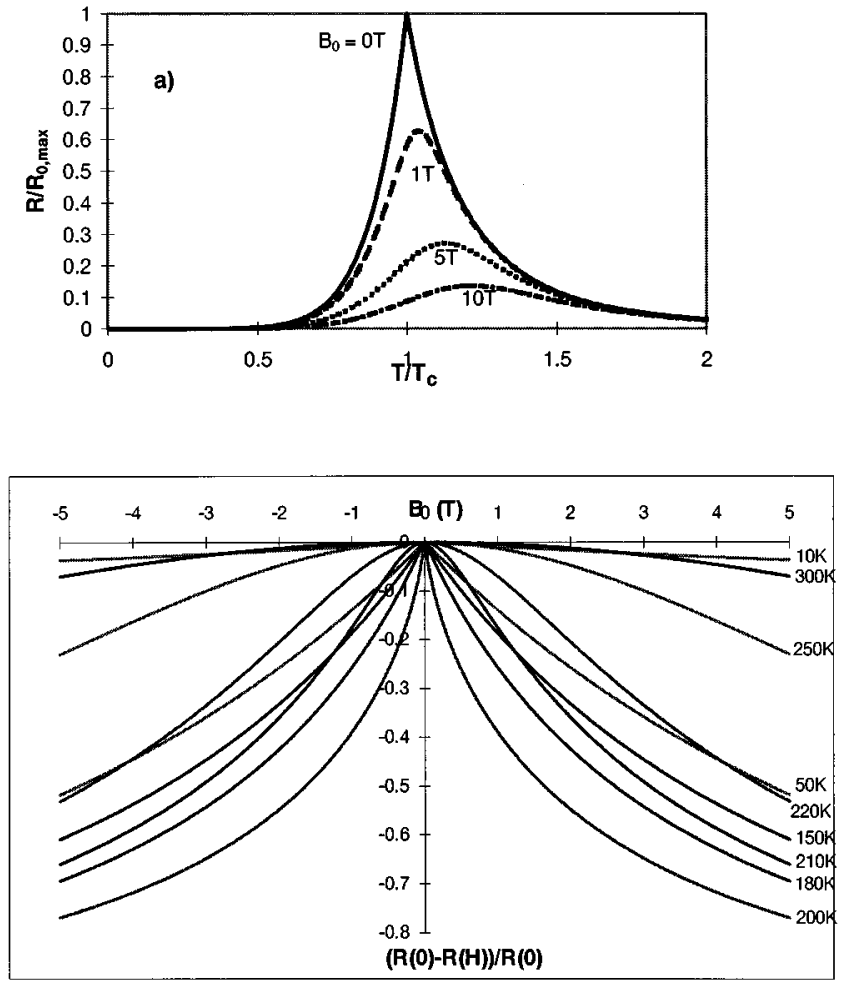

FIG. 3. Resistivity variations at (a) constant applied fields and (b) constant temperatures with Brillouin $S=3 / 2$ magnetization $\left(T_{C}=200 \mathrm{~K}\right.$ and $\left.T_{0}=4 \times 10^{7} \mathrm{~K}\right)$.

(=10 Dq) is the octahedral crystal-field splitting of the ion, which is estimated from the energy of the ${ }^{5} E_{g} \rightarrow{ }^{5} T_{2 g}$ optical transition as roughly $2 \mathrm{eV} .^{24,25}$ That $U_{m}$ is not greatly different from $\Delta$ is evidenced by the fact that $\mathrm{Co}^{3+}$ is $\mathrm{LaCoO}_{3}$ is in a low-spin state. ${ }^{26}$ A recent estimate from optical spectra is $U_{m} \approx 2 \mathrm{eV}^{27}$

In estimating the density of states to include in Eq. (3), the probability of Eq. (4) must be multiplied by the number of available states $/ \mathrm{m}^{3}$. Hence,

$$
N\left(E_{m}\right)=p\left(E_{m}\right)(1-x) \phi g / v,
$$

where $(1-x)=0.3$ is the probability that the $e_{g}$ orbital at the manganese site receiving the hopping electron is unoccupied, $\phi$ is a geometric factor of order 0.5 which accounts for the fact that the hopping electron has a $d_{z 2}$ rather than an $s$-state wave function, ${ }^{14}$ and $g$ is the probability that an unoccupied manganese orbital can actually accept an electron. That $g \neq 1$ is a reflection of the dynamic Jahn-Teller effect. When $\Delta E$ is less than the Jahn-Teller stabilization energy $(\approx 0.5 \mathrm{eV})$ associated with the tetragonal extension of the octahedron necessary to accommodate a $d_{z 2}$ electron, a hop can only take place if the receiving site is free to distort or is already suitably distorted. The problem is illustrated in two dimensions in Fig. 2. When $x=0.3$, the factor $g$ in three dimensions is approximately 0.7 . Hence the value of $N\left(E_{m}\right)$ deduced from Eqs. (4) and (5) is 9 $\times 10^{26} \mathrm{~m}^{-3} \mathrm{eV}^{-1}$. In terms of $T_{0}$, Eq. (3) becomes $k T_{0}$ $=171 \alpha^{3} U_{m} v$. Taking $U_{m}=2 \mathrm{eV}$, the corresponding localization lengths for $\left(\mathrm{La}_{0.7} \mathrm{Sr}_{0.3}\right) \mathrm{MnO}_{3}$ and $\left(\mathrm{Pr}_{0.7} \mathrm{~Pb}_{0.3}\right) \mathrm{MnO}_{2}$ are 0.45 and $0.08 \mathrm{~nm}$, respectively, and the average hopping

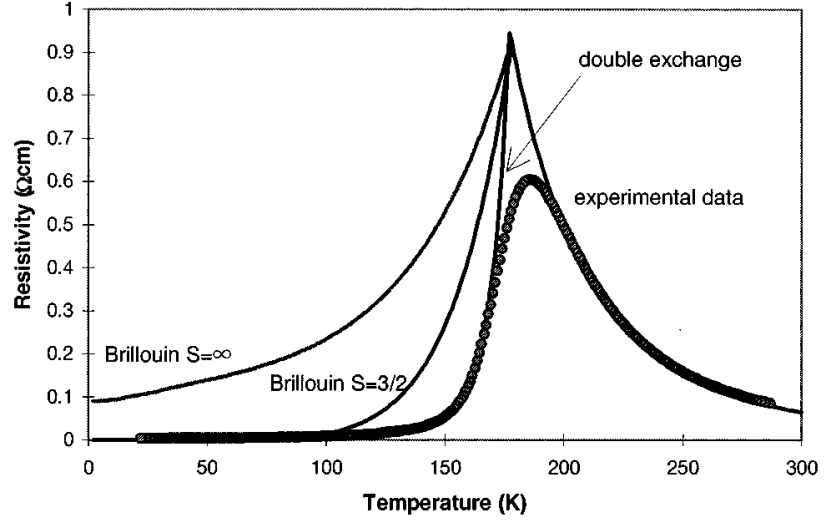

FIG. 4. Comparison of the magnetic localization model with data measured on a good-quality $\mathrm{La}_{0.7} \mathrm{Ca}_{0.3} \mathrm{MnO}_{3}$ thin film. The three curves are fits obtained using magnetizations from a Brillouin function with $S=3 / 2$ and $S=\infty$ (large spin clusters) and the doubleexchange model.

ranges at room temperature are 1.64 and $1.06 \mathrm{~nm}$. The hopping energy at room temperature is $\Delta E \approx 0.1 \mathrm{eV}$. These numbers are physically plausible since the localization length exceeds the ionic radius of $\mathrm{Mn}^{3+}$ and the hopping distances are 3-4 times the Mn-Mn separation.

When a magnetic field is applied to the manganite or when there is an internal molecular field, the random distribution of spin directions is narrowed and $p\left(E_{m}\right)$ is modified. The average potential is of the form

$$
\left\langle E_{m}\right\rangle=\frac{1}{2} U_{m}\left(1-\left\langle\cos \theta_{i j}\right\rangle\right) .
$$

If the azimuthal angle $\phi_{i}$ is randomly distributed and if $\theta_{i}$ is uncorrelated, then by averaging over $\phi_{i}$ it can be shown that $\left\langle\cos \theta_{i j}\right\rangle=\left\langle\cos \theta_{i}\right\rangle^{2}$, where $\theta_{i}$ is the angle the spins make with the applied field. The local magnetization $M$ can also be expressed as a function of $\theta_{i}$ by $M=M_{S}\left\langle\cos \theta_{i}\right\rangle$, where $M_{S}$ is the saturation magnetization. In the approximation of a square distribution of single-electron potentials with no short-range correlations, the potential width becomes $U_{m}\left\{1-\left(M / M_{S}\right)^{2}\right\}$ and we obtain, for $T_{0}$,

$$
\begin{aligned}
k T_{0} & =18 \alpha^{3}\left[U_{m}\left\{1-\left(M / M_{S}\right)^{2}\right\} v /(1-x) \phi g\right] \\
& =171 \alpha^{3} U_{m}\left\{1-\left(M / M_{S}\right)^{2}\right\} v
\end{aligned}
$$

where $M_{S}$ is the collinear saturation.

Taking the temperature variation of $M$ from molecular field theory, we obtain the temperature variation of the resistivity from Eq. (7). Three cases are of particular interest. One is Brillouin theory with $S=3 / 2$, another is the Langevin expression for large classical spins, and the last one is the double-exchange theory developed by Kubo and Ohata. ${ }^{28}$ The calculated temperature and field variations of the resistivity are shown in Fig. 3. Here $T_{C}$ is taken as $200 \mathrm{~K}$ and $171 \alpha^{3} U_{m} v=4 \times 10^{7} \mathrm{~K}$. These curves demonstrate that the magnetic localization model is able to reproduce the experimental behavior of the mixed-valence manganites with $x$ $=0.3$. For the higher- $T_{C}$ compounds where there is little spin canting in the ferromagnetic case, the main problem is to describe the dramatic decrease of $\rho$ just below $T_{C}$. Figure 4 
is an attempt to fit the resistivity of a good-quality $\left(\mathrm{La}_{0.7} \mathrm{Ca}_{0.3}\right) \mathrm{MnO}_{3}$ thin film with our model using different expressions for the magnetization. Except at $T_{C}$, where short-range magnetic correlations should be considered, the agreement with the data is excellent when using Kubo and Ohata's magnetization, which has been shown to apply to perfect double-exchange compounds. ${ }^{28}$ Another interesting feature of the model is that the residual resistivity $\rho_{0}$ is zero for any quantum spin provided that the ferromagnetism is saturated, but for large classical spins (Langevin theory) or if there is a residual spin canting at $T=0$ so that $M / M_{S} \neq 1$, the value of $\rho_{0}$ is a significant fraction of the peak resistivity. Figure 4 numerically demonstrates that large classical spin clusters lead directly to an enhanced $\rho_{0}$. In the light of heat capacity measurements, we think the vision of a magnetically homogeneous medium at low temperature may be erroneous. We previously attributed high residual resistivity to transport impeded by the canted spins at the surface of large ferromagnetic spin packets, and these entities are now being studied by small-angle neutron scattering. ${ }^{29}$

The theory of magnetic localization presented here could be refined by considering magnetic correlations over the hopping distance $(\approx 1 \mathrm{~nm})$ instead of the mean magnetization. The Jahn-Teller effect can also be better taken into account by a more elaborate treatment of the factor $g$ in Eq. (5), but these are details. The essential physics of the metalinsulator transitions is localization associated with magnetic disorder.
*Present address: CE Saclay, DRECAM/SPEC, 91191 Gif sur Yvette, France.

${ }^{1}$ J. M. D. Coey, M. Viret, and S. von Molnar, Adv. Phys. (to be published).

${ }^{2}$ M. Kasai, Y. Kanke, T. Ohno, and Y. Kozono, J. Appl. Phys. 72, 534 (1992).

${ }^{3}$ R. von Helmholt, J. Wecker, B. Holzapfel, L. Schultz, and K. Samwer, Phys. Rev. Lett. 71, 2331 (1993).

${ }^{4}$ G. C. Xiong, Q. Li, H. L. Ju, S. N. Mao, L. Senapati, X. X. Xi, R. L. Greene, and T. Venkatesen, Appl. Phys. Lett. 66, 1427 (1995); G. C. Xiong, Q. Li, H. L. Ju, R. L. Greene, and T. Venkatesen, ibid. 66, 1689 (1995).

${ }^{5}$ H. Y. Hwang, S. W. Cheong, P. G. Radaelli, M. Marezio, and B. Batlogg, Phys. Rev. Lett. 75, 914 (1995).

${ }^{6}$ C. Zener, Phys. Rev. 81, 440 (1950); 82, 403 (1950).

${ }^{7}$ J. B. Goodenough, Phys. Rev. 100, 564 (1955).

${ }^{8}$ D. Sarma, N. Shanthi, S. Barman, N. Hamada, H. Sawada, and K. Terakura, Phys. Rev. Lett. 75, 1126 (1995).

${ }^{9}$ A. J. Millis, P. B. Littlewood, and B. I. Shraiman, Phys. Rev. Lett. 74, 5144 (1995).

${ }^{10}$ Y. X. Jia, Li Lu, K. Khazeni, V. H. Crespi, A. Zettl, and M. L. Cohen, Phys. Rev. B 52, 9147 (1995).

${ }^{11}$ M. F. Hundley, J. J. Neumeier, R. H. Heffner, Q. X. Jia, X. D. Wu, and J. D. Thompson, J. Appl. Phys. 79, 4535 (1996).

${ }^{12}$ J. Nunez-Regueiro and A. Kadin, Appl. Phys. Lett. 68, 2747 (1996).

${ }^{13}$ J. M. D. Coey, M. Viret, L. Ranno, and K. Ounadjela, Phys. Rev. Lett. 75, 3910 (1995).

${ }^{14}$ N. F. Mott, Metal-Insulator Transitions, 2nd ed. (Taylor \& Francis, London, 1990).

${ }^{15}$ A. Gupta, G. Q. Gong, Gang Xiao, P. R. Duncombe, P. Lecoeur,
P. Trouilloud, Y. Y. Wang, V. P. Dravid, and J. Z. Sun, Phys. Rev. B 54, 15629 (1996).

${ }^{16}$ G. Snyder, R. Hiskes, S. DiCarolis, M. Beasley, and T. Geballe, Phys. Rev. B 53, 1 (1996).

${ }^{17}$ Y. X. Jia, Li Lu, K. Khazeni, D. Yen, C. S. Lee, and A. Zettl, Solid State Commun. 94, 917 (1995).

${ }^{18}$ P. Wagner, V. Metlushko, L. Trappeniers, A. Vantomme, J. Vanacken, G. Kido, V. Moshchalkov, and Y. Bruynseraeded, Phys. Rev. B (to be published).

${ }^{19}$ A. L. Efros and B. L. Shklovskii, J. Phys. C 8, L49 (1975).

${ }^{20}$ L. Ranno, M. Viret, J. M. D. Coey, and K. Ounadjela (unpublished).

${ }^{21}$ N. F. Mott and E. A. Davies, Electronic Processes in Noncrystalline Solids, 2nd ed. (Oxford University Press, New York, 1979); H. Boettger and V. Bryksin, Hopping Conduction in Solids (Akademic-Verlag, Berlin, 1985).

${ }^{22}$ V. Ambegaokar, B. I. Halperin, and J. S. Langer, Phys. Rev. B 4, 2612 (1971).

${ }^{23}$ J. H. Park, C. T. Chen, W. W. Cheong, W. Bao, G. Meigs, V. Chakarian, and Y. U. Idzerda, Phys. Rev. Lett. 76, 4215 (1996).

${ }^{24}$ G. Subba Rao, C. Rao, and J. Ferraro, Appl. Spectrosc. 24, 436 (1970).

${ }^{25}$ J. F. Lawler, J. G. Lunney, and J. M. D. Coey, Appl. Phys. Lett. 65, 3017 (1994)

${ }^{26}$ J. B. Goodenough, Magnetism and the Chemical Bond (Interscience, New York, 1963).

${ }^{27}$ Y. Okimoto, T. Katsufuji, T. Ishikawa, A. Urushibara, T. Arima, and Y. Tokura, Phys. Rev. Lett. 75, 109 (1995).

${ }^{28}$ K. Kubo and N. Ohata, J. Phys. Soc. Jpn. 33, 21 (1972).

${ }^{29}$ M. Viret, H. Glattli, C. Fermon, L. Ranno, and J. M. D. Coey (unpublished). 